\title{
Compte-rendu de la séance du 22 novembre 1986
}

La Société Beige de Dermatologie et de Syphiligra-phie s'est réunie au campus du Sart-Tilman de l'Uni-versité de Liège, à Tinvitation du Professeur CM. Lapière.

La matinee est consacrée à la presentation des cas cliniques et des communications suivantes: Delvoye, P.; Ghazi, A.; Piérard, G.E.; Lapière, CM.: Vascularite granulomateuse: discussion étiolo-gique

Delvoye, P.; Neys, C; Ghazi, A.; Piérard, G.E.; Lapière, CM.: Vascularite à éosinophiles et escarres de décubitus

Gedeah, G.; Darcis, J.M.; Piérard, G.E.; Lapière, CM.: Vascularite leucocytoclasique d'origine infec-tieuse

Gedeah, G.; Darcis, J.M.; Piérard, G.E.; Lapière, CM.: Reactions cutanéo-muqueuses aux sels d'or

Dooms-Goossens, A.; Degreef, H.; Bourgeois, M.; Goelen, M.: Contactallergie op schoenen Hermanns, J.F.; Piérard, G.E.: Halogénides végé-tantes

Hermanns, J.F.; Lother, Y; Legros, J.J.; Piérard, G.E.: Lipomatose de Launois-Bensaude-

Madelung

Rurangirwa, A.; Van Cauwenberghe, D.: Lipodys-trophie partielle de Barraquer-Simons

Mélotte, P.; Damseaux, M.; Piérard-Franchimont, C; Piérard, G.E.: La liposuction

Dehavay, J.: Varicelle néonatale

Dehavay, J.: Epidermolyse bulleuse simple léthale

Soyeur-Broux, M.; de la Brassinne, M.; Cornil, F.; Sadzot, B.; Piérard, G.E.: Angioendothéliomatose proliférative systémique maligne

de la Brassinne, M.; Soyeur-Broux, M.; Cornil, F; Soumagne, D.; Piérard, G.E.: Sarcome de Kaposi des membres supérieurs

de la Brassinne, M.; Dethier, F; Piérard, G.E.; Lapière, CM.: Hyperplasie angiolymphoïde avec éosi-nophilie

Society Proceedings

207

Delvoye, P.; Le, T.; Franchimont, C; Dehavay, J.; Piérard, G.E.; Lapière, M.: Lymphome T de haute malignité: du rapport avec le HTLV-1 et de la thérapeu-tique

Le, T.; Lother, Y.; Ghazi, Y.; Piérard, G.E.; Lapière, CM.: Mycosis fungoïde: Traitement par badigeon-nage à l'ypérite

Le, T.; Piérard, G.E.; Piérard-Franchimont, C: Immunocytome lymphoplasmocytoïde et sarcoïde Constandt, G.; Kint, A.: Dermatitis herpetiformis van Duhring met minimale symptomatologie Constandt, G.; Oomen, C.; Duprez, D.; Kint, A.: Urticaria pigmentosa met systeemmastocytose Darcis, J.M.; Foidart, J.M.; Gielen, D.; Piérard, G.E.: Herpes gestationis

Darcis, J.M.; Delvoye, P.; Piérard, G.E.; Lapière, CM.: Syphilis secondaire et gale

Delvoye, P.; Ghazi, A.; Darcis, J.M.; Piérard, G.E.; Lapière, CM.: Ulcère de Marjolin: discussion noso-graphique à propos de deux cas 
de la Brassinne, M.; Piérard-Franchimont, C; Sproten, C; Piérard, G.E.: Maladie de Paget extramammaire

Cornil, F; de la Brassinne, M.; Soyeur-Broux, M.: Tumeur de Merkel

Dehavay, J.: Ichtyose acquise paranéoplasique

Gedeah, G.: Anévrisme veineux dans la maladie de von Recklinghausen

Gedeah, G.; Piérard; G.E.; Lapière, CM.: Psoriasis et hypertrichose

Les deux themes étudiés $\Gamma$ après-midi sont la patho-logie des follicules sébacés et la maladie du greffon

contre Thôte (MGCH), avec les communications sui-vantes:

Lapière, CM.: Introduction à la pathologie des glandes sébacées

Piérard-Franchimont, C; Piérard, G.E.: Séborrhée et calvitie

Saint-Léger, D.: Composition du sebum et acné

Piérard, G.E.; Le, T.: Séborrhée, acné et leurs traite-ments

Saurat, J.H.: Mode d'action des rétinoïdes

Piérard, G.E.; Piérard-Franchimont, C; Hassou-nah, A.; Lother, Y.: Vieillissement des glandes

sébacées et maladie de Favre-Racouchot

Lapière, CM.: La maladie du greffon contre Thôte: Introduction

Bury, J.: Greffe de moelle osseuse (GMO): Aspects cliniques

Saurat, J.H.: Formes cliniques de la MGCH

Lapière, CM.: La MGCH chronique, modèle de sclérose du derme

de la Brassinne, M.: Photochimiothérapie de la GVHR cutanée chronique

Piérard, G.E.; Nickels-Read, D; Piérard-Franchimont, C: Pronostic et diagnostic différentiel de la maladie du greffon contre Thôte traitée par cyclospo-rine

Elections: Les Drs Bourgeois, Candaele, Ceuterick, Chrispeels, Nop et Vanderheyden sont élus membres-associés. 